J.N. Coleman, Liquid exfoliation of defect free graphene, Accounts of Chemical Research

\title{
Liquid exfoliation of defect-free graphene
}

Jonathan N Coleman

School of Physics and CRANN, Trinity College Dublin, Dublin 2, Ireland

colemaj@tcd.ie

\section{Conspectus}

Due to its unprecedented physical properties, graphene has generated huge interest over the last 7 years. Graphene is generally fabricated in one of two ways: as very high quality sheets produced in limited quantities by micromechanical cleavage or vapour growth, or a rather defective, graphenelike material, graphene oxide, produced in large quantities. However, a growing number of applications would profit from the availability of a method to produce high quality graphene in large quantities.

This Account describes recent work to develop such a processing route inspired by previous theoretical and experimental studies on the solvent-dispersion of carbon nanotubes. That work had shown that nanotubes could be effectively dispersed in solvents whose surface energy matched that of the nanotubes. We describe the application of the same approach to the exfoliation of graphite to give graphene in a range of solvents. When graphite powder is exposed to ultrasonication in the presence of a suitable solvent, the powder fragments into nanosheets, which are stabilized against aggregation by the solvent. The enthalpy of mixing is minimized for solvents with surface energies close to that of graphene $\left(\sim 68 \mathrm{~mJ} / \mathrm{m}^{2}\right)$. The exfoliated nanosheets are free of defects and oxides and can be produced in large quantities. Once solvent exfoliation is possible, the process can be optimized and the nanosheets can be separated by size. The use of surfactants can also stabilize exfoliated graphene in water, where the zeta potential of the surfactant-coated graphene nanosheets controls the dispersed concentration.

Liquid exfoliated graphene can be used for a range of applications: graphene dispersions as optical limiters, films of graphene flakes as transparent conductors or sensors, and exfoliated graphene as a mechanical reinforcement for polymer-based composites. Finally, we have extended this process to exfoliate other layered compounds such as $\mathrm{BN}$ and $\mathrm{MoS}_{2}$. Such materials will be important in a range of applications from thermoelectrics to battery electrodes. This liquid exfoliation technique can be applied to a wide range of materials and has the potential to be scaled up into an industrial process. We believe the coming decade will see an explosion in the applications involving liquid exfoliated two dimensional materials. 


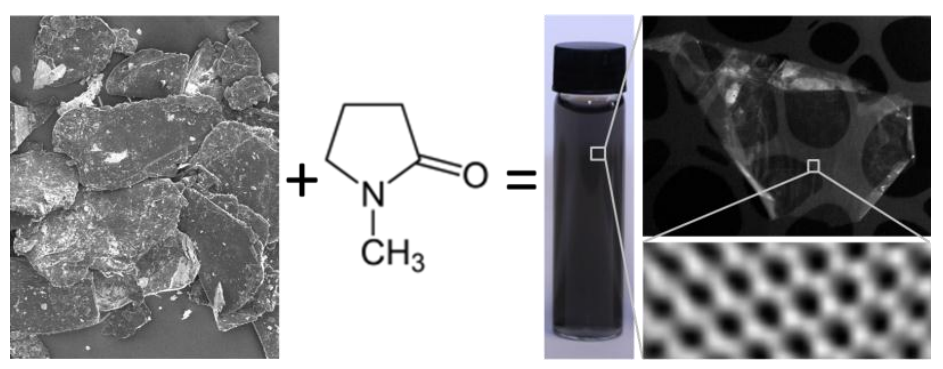

\section{Introduction}

Graphite has been used by man for thousands of years, originally for decorative purposes, ${ }^{1}$ but later in a range of applications from pencils to lining moulds for cannon balls. While it had long been known that graphite had a stratified nature, the details of its layered structure were only revealed by Bernal in 1924 using X-ray crystallography. ${ }^{2}$ We now know that graphite is a crystal consisting of stacked carbon monolayers which are known individually as graphene. ${ }^{3,4}$ A graphene sheet consists of an atomically thin array of $\mathrm{sp}^{2}$ bonded carbon atoms organized in a planar hexagonal arrangement. Studied theoretically for many years, ${ }^{5}$ thermodynamic arguments suggested that graphene could not exist as a free-standing entity. ${ }^{6}$ Nevertheless, over the years a number of groups attempted to produce extremely thin graphitic lamellae. ${ }^{7,8}$ This work culminated in 2004 with the discovery by Geim and Novosolov that individual sheets of graphene could be obtained by micromechanical cleavage of graphite. ${ }^{9}$

The novel electronic properties of graphene have been well documented. ${ }^{4}$ In addition, graphene is ideal for a range of applications. For example, it is the strongest material known to $\operatorname{man}^{10}$ and has been fabricated into large area transparent conductors. ${ }^{11}$ Because of these exciting properties, a number of new methods of graphene production have been developed such as by annealing $\mathrm{SiC}$ substrates ${ }^{12}$ or growth on metal supports. ${ }^{13,14}$

These methods for producing graphene have been very successful to date. However, it is likely that many future industrial applications of graphene will be in sectors such as large-area coatings or composite fillers which require the production of graphene in very large quantities. ${ }^{15}$ To this end, it is likely that liquid phase production methods will be required. ${ }^{16}$

Recently, a large number of papers have described the dispersion and exfoliation of graphene oxide (GO). ${ }^{17}$ This material consists of graphene-like sheets, chemically functionalised with groups such as hydroxyls and epoxides, which stabilise the sheets in water ${ }^{17}$. Graphene oxide has been an extremely successful material and has led to a string of advances. ${ }^{18}$ However, the functionalisation results in considerable disruption of the electronic structure of the graphene. In fact GO is an insulator rather than a semi-metal and is conceptually different from graphene. While the functionalities can be removed by reduction, large defect populations remain, which continue to 
J.N. Coleman, Liquid exfoliation of defect free graphene, Accounts of Chemical Research

disrupt the electronic properties. ${ }^{17}$ Thus, for a range of applications where pristine graphene is required, a non-covalent, solution-phase method to produce significant quantities of defect free, unoxidised graphene would be extremely useful.

Liquid exfoliation of graphite to give graphene

Genesis

For a number of years before the popularisation of graphene, a number of groups studied dispersion and exfoliation of carbon nanotubes in solvents. ${ }^{19}$ In 2008 , Bergin et al combined experiment and thermodynamic modelling to show that the enthalpy of mixing of nanotubes per volume of solvent is given by

$$
\Delta H_{m i x} / V \approx 4\left(\sqrt{E_{S, N T}}-\sqrt{E_{S, S o l}}\right)^{2} \phi / D
$$

where $\phi$ is the dispersed nanotube volume fraction, $D$ is the dispersed nanotube (or bundle) diameter and $E_{S, N T}$ and $E_{S, S o l}$ are the nanotube and solvent surface energies respectively. This suggested successful solvents to be those with surface energy close to that of the nanotubes. ${ }^{20,21}$ Recently, we showed that the maximum dispersible concentration of rigid rods in solvents is given by: ${ }^{22}$

$$
\phi \approx K^{\prime} \exp \left[-\frac{\bar{v}}{R T} \frac{\partial\left(\Delta H_{M i x} / V\right)}{\partial \phi}\right]
$$

where $K^{\prime}$ is a constant and $\bar{v}$ is the molar volume of rods. Applying to nanotubes by inserting (1) into (2) gives

$$
\phi \approx K^{\prime} \exp \left[-\frac{\pi D L}{4 E_{S, N T} k T}\left(E_{S, N T}-E_{S, S o l}\right)^{2}\right]
$$

where $\mathrm{L}$ is the mean nanotube length. This expression represents a Gaussian function when $\phi$ is plotted versus the solvent surface energy. Experimental data agrees very well with this model confirming that nanotubes can be dispersed at high concentration once the solvent and nanotube surface energies match. ${ }^{22}$

\section{Exfoliation of graphene in solvents}

Once the role of surface energy was known it became immediately clear that, as graphite has a surface energy similar to carbon nanotubes, it might be possible to exfoliate graphite to give graphene in certain solvents. To test this, we sonicated powdered graphite in a range of solvents using a low power sonic bath. ${ }^{23}$ We initially focused on solvents known to exfoliate nanotubes such as N-methyl-pyrrolidone (NMP) and dimethylformamide (DMF) (figure 1A, inset). After centrifuging to remove any undispersed material, we measured the dispersed concentration using a 
J.N. Coleman, Liquid exfoliation of defect free graphene, Accounts of Chemical Research

combination of filtration, weighing and absorption spectroscopy. We found the dispersed concentration peaked for solvents with surface tensions close to $40 \mathrm{~mJ} / \mathrm{m}^{2}$, which is equivalent to surface energies of $\sim 70 \mathrm{~mJ} / \mathrm{m}^{2}$ (figure 1A). ${ }^{24}$ To understand this, we calculated the enthalpy of mixing for graphene flakes dispersed and exfoliated in solvents to be:

$\frac{\Delta H_{M i x}}{V} \approx \frac{2}{T_{N S}}\left(\sqrt{E_{S, S}}-\sqrt{E_{S, G}}\right)^{2} \phi_{G}$

where $E_{S, S}$ and $E_{S, G}$ are the solvent and graphene surface energy respectively, $\mathrm{T}_{\mathrm{NS}}$ is the nanosheet thickness and $\phi_{\mathrm{G}}$ is the dispersed graphene volume fraction. Assuming we can use equation 2 to describe dispersed platelets, this predicts the dispersed graphene concentration $\left(C_{G} \propto \phi_{G}\right)$ should be described by:

$$
C_{G} \propto \exp \left[-\frac{\pi D_{G}^{2}}{8 E_{S, G} k T}\left(E_{S, S}-E_{S, G}\right)^{2}\right]
$$

where $D_{G}$ is the diameter of a graphene sheet (modelling it as a disc). We find this model fits the data extremely well (dashed line), suggesting a surface energy of graphene of $\sim 68 \mathrm{~mJ} / \mathrm{m}^{2}$. While a wide range of values have been reported for the surface energy of graphite, this is consistent with a recent value of $53 \mathrm{~mJ} / \mathrm{m}^{2}$, obtained from contact angle measurements. ${ }^{25}$

We analysed the exfoliation state of the dispersed material in a number of solvents using transmission electron microscopy. We found large quantities of 2-dimensional objects which appeared from careful examination of their edges ${ }^{26}$ to be either monolayers (figure 1B) or multilayers (figure 1C). We can definitively identify the presence of monolayers by electron diffraction. ${ }^{27}$ Shown in figures 1D\&E are electron diffraction patterns taken from what appear to be a graphene monolayer and a graphene bi-layer respectively. Computational studies have shown that the inner set of spots is more intense for monolayers and vice versa for multilayer graphene. ${ }^{28}$ This is exactly what is found here, confirming our visible identification. Using a combination of electron diffraction and inspection, we can estimate the number of monolayers in each flake observed by TEM. As shown in figure $1 \mathrm{~F}$, we found approximately $25 \%$ monolayers with the vast majority of flakes having $<5$ layers. $^{23}$

While it is clear that graphite can be exfoliated in solvents to give mono- and few layer graphene, it is not yet clear if the graphene is pristine. To test this, we performed extensive Raman characterisation of our exfoliated material. ${ }^{23}$ Typically we observe spectra such as the upper one in figure $1 \mathrm{G}$, with a well-defined $\mathrm{D}$ band and a broad 2D band, characteristic of few layer flakes. ${ }^{29}$ However, a D band around $1300 \mathrm{~cm}^{-1}$ is also observed, indicative of defects. It is worth noting that for flakes in the size range here, the edges will contribute to the spectrum, appearing as defects. ${ }^{29}$ To 
J.N. Coleman, Liquid exfoliation of defect free graphene, Accounts of Chemical Research

test this, we identified a flake with dimensions significantly larger than the laser spot. Its spectrum is the lower one in figure $1 \mathrm{G}$ and clearly displays no D band. Measurements such as these have clearly demonstrated that the flakes produced using solvent exfoliation are relatively defect-free. It is also important to confirm that no oxidation of the flakes has occurred during processing. We have performed X-ray photoelectron spectroscopy on thin films of graphene flakes as shown in figure $1 \mathrm{H}$. The spectrum is dominated by the C-C peak. While some very small additional peaks are observed between 385 and $287 \mathrm{eV}$, these are associated with residual solvent.

This data makes it clear that solvents with the correct surface energy can exfoliate graphite to give reasonable quantities of high quality defect-free graphene. However, surface energy is not an ideal predictor of solvent quality i.e. not all solvents with surface energy close to $68 \mathrm{~mJ} / \mathrm{m}^{2}$ exfoliate graphene. The reason for this is that surface energy is a rather unsophisticated solubility parameter. More useful are the Hansen Solubility parameters. ${ }^{30}$ These are known to describe the dispersion of nanotubes well ${ }^{21}$ suggesting them to be appropriate for use with graphene dispersions. The Hansen solubility parameters are the square roots of the dispersive, polar and hydrogen bonding components of the cohesive energy density of a material and are denoted $\delta_{\mathrm{D}}, \delta_{\mathrm{P}}$ and $\delta_{\mathrm{H}}$ respectively. Hansen has suggested that the enthalpy of mixing is given by ${ }^{30}$

$$
\frac{\Delta H_{M i x}}{V} \approx\left[\left(\delta_{D, S}-\delta_{D, G}\right)^{2}+\left(\delta_{P, S}-\delta_{P, G}\right)^{2} / 4+\left(\delta_{H, S}-\delta_{H, G}\right)^{2} / 4\right] \phi_{G}
$$

where the subscripts $S$ and $G$ represent solvent and graphene respectively. Substituting this expression into (2) gives an expression for the dispersed concentration, $\mathrm{C}_{\mathrm{G}}$ :

$$
C_{G} \propto \exp \left[-\frac{\bar{v}_{G}}{R T}\left(\delta_{D, S}-\delta_{D, G}\right)^{2}\right] \exp \left[-\frac{\bar{v}_{G}}{R T}\left(\delta_{P, S}-\delta_{P, G}\right)^{2} / 4\right] \exp \left[-\frac{\bar{v}_{G}}{R T}\left(\delta_{H, S}-\delta_{H, G}\right)^{2} / 4\right]
$$

Where $\bar{v}_{G}$ is the graphene molar mass. This predicts that the dispersed concentration is maximised in solvents where all Hansen parameters match those of graphene. This is shown to be the case in figure $2,{ }^{31}$ which shows well-defined peaks bounded by Gaussian functions (equation 7). This data suggests that graphene is described by Hansen parameters of $\delta_{\mathrm{D}, \mathrm{G}} \approx 18 \mathrm{MPa}^{1 / 2}, \delta_{\mathrm{P}, \mathrm{G}} \approx 10 \mathrm{MPa}^{1 / 2}$, and $\delta_{\mathrm{H}, \mathrm{G}} \approx 7$ $\mathrm{MPa}^{1 / 2}$.

We note that equation 7 can be abbreviated to

$$
C_{G}=\exp \left[\bar{v}_{G} \chi / \bar{v}_{S}\right]
$$

where $\bar{v}_{S}$ is the solvent molar mass and $\chi$ is the Flory-Huggins parameter. This parameter is can be estimated from the graphene and solvent Hansen parameters (equations 7 and 8) and is often used to discuss the energetics of polymer solutions. Equation 8 predicts that $\ln C_{G}$ should fall linearly with $\chi$. The inset in figure 2 shows this predicted behaviour, supporting the idea that solution 
J.N. Coleman, Liquid exfoliation of defect free graphene, Accounts of Chemical Research

thermodynamics can be used as a framework to describe graphene-solvent mixtures. It is worth noting that during this work, ${ }^{31}$ early analysis of the data for a subset of solvents suggested approximate Hansen parameters for graphene. This allowed us to search for new solvents with Hansen parameters in this region. This approach resulted in the discovery of a number of new solvents for graphene. However, it is worth emphasising that this does not mean that the analysis described above is a complete description of the dispersion process. It is doubtful whether the combination of dispersive, polar and H-bonding interactions accurately describes the system at the molecular scale. More work is required to fully understand graphene-solvent interactions.

The concentrations shown in figures $1 \mathrm{~A}$ and 2 are too low to be useful for many applications. We attempted to increase the dispersed concentration by optimising the processing parameters, notably the sonication time. We prepared dispersions of graphene in NMP which were bath-sonicated for a range of times from 0.5 to $\sim 400$ hours. ${ }^{26}$ Using absorption spectroscopy to measure the dispersed concentration after centrifugation, we found $\mathrm{C}_{\mathrm{G}}$ to increase from $0.05 \mathrm{mg} / \mathrm{ml}$ to $>1 \mathrm{mg} / \mathrm{ml}$ (figure $3 \mathrm{~A}$ ). Interestingly, this graph showed $\mathrm{C}_{\mathrm{G}}$ to scale very well with the square root of sonication time: $\mathrm{C}_{\mathrm{G}} \propto \sqrt{ }_{\mathrm{t}}$. TEM characterisation showed the flakes to appear of good quality, even after long sonication times, with on average $\sim 3$ monolayers per flake for all sonication times. However, the mean flake length, $\langle\mathrm{L}\rangle$, and width, $\langle\mathrm{w}\rangle$, were observed to fall as $\mathrm{t}^{-1 / 2}$ as predicted for sonication induced scission. ${ }^{32}$ Detailed analysis shows that this time dependence leads to the observed $\mathrm{C}_{\mathrm{G}} \propto \sqrt{t}_{\mathrm{t}}$ behaviour. $^{26}$

It is worth considering whether the long sonication induces basal plane defects. While Raman spectra of films deposited from the dispersed graphene showed a D band, it is very small compared to defective graphene-like materials. ${ }^{17}$ This suggests the $\mathrm{D}$ band to be associated with flake edges. Were this the case, the average ratio of Raman D to G bands should scale as the flake edge to area ratio: $\left\langle I_{D} / I_{G}\right\rangle \propto\left[\langle L\rangle^{-1}+\langle w\rangle^{-1}\right]$. We measured this ratio and the mean flake length and width using TEM. $^{26}$ As shown in figure $3 \mathrm{~B}$, we see very good agreement with this prediction. This confirms that the Raman data is entirely consistent with the production of edges by sonication, suggesting that the flakes are largely free of point defects. In addition, by fitting the data we found that

$$
\left\langle I_{D} / I_{G}\right\rangle(t)-\left(I_{D} / I_{G}\right)_{p o w d e r}=\Delta I_{D} / I_{G}=0.065\left[\langle L\rangle^{-1}+\langle w\rangle^{-1}\right]
$$

where the dimensions are measured in microns. ${ }^{26}$ From the TEM analysis we know that $\langle L / w\rangle \sim 3$, allowing us to approximate: $\Delta I_{D} / I_{G} \approx 0.26 /\langle L\rangle$. This expression can be used to estimate the size of flakes from Raman measurements. 
J.N. Coleman, Liquid exfoliation of defect free graphene, Accounts of Chemical Research

While concentrations of $\sim 1 \mathrm{mg} / \mathrm{ml}$ can be achieved by long sonication, it would be useful to push this even higher if possible. We found that if a graphene dispersion, prepared as described above, is filtered through a membrane to give a film, it retains the Raman spectrum of few layer flakes $^{29}$ rather than reaggregating to graphite. ${ }^{26}$ This suggests that within the film, the flakes are weakly interacting and so should be easy to redisperse. We found this to be the case; sonication of such restacked films, gave dispersed concentrations of up to $60 \mathrm{mg} / \mathrm{ml} .^{33}$ While these dispersions were unstable, we found that $\sim 25 \mathrm{mg} / \mathrm{ml}$ remained dispersed indefinitely.

Another problem with solvent dispersed graphene is that the flake length is typically $<1 \mu \mathrm{m}$, too small for many applications such as mechanical reinforcement of composites. ${ }^{34}$ To address this we attempted to select flakes by size, separating large from small. ${ }^{18}$ By centrifuging a high concentration dispersion at $4000 \mathrm{rpm}$, we separated the smallest flakes into the supernatant from the larger ones in the sediment. The sediment was redispersed and centrifuged at $3000 \mathrm{rpm}$. This again separated smaller (supernatant) from larger flakes (sediment). This procedure was repeated a number of times until a centrifugation rate of $500 \mathrm{rpm}$ was reached. We characterised the flake size in each supernatant by TEM and using Raman spectroscopy. We round the flake size to increase from $\sim 1 \mu \mathrm{m}$ for the $4000 \mathrm{rpm}$ sample to $\sim 3 \mu \mathrm{m}$ for the $500 \mathrm{rpm}$ sample (figure 3C). This method is both practical and effective and is now in standard usage in our lab to prepare dispersions of relatively large flakes.

A final problem with our approach to dispersing graphene is that solvents which have the correct surface energy to disperse graphene also have high boiling points, causing practical difficulties. By examining the data in figure 2 more closely, we found a small number of solvents, notably isopropanol and acetone, with relatively low boiling points. Unfortunately, they dispersed graphene at concentrations much lower than the better solvents. To address this we used the approach described above of increasing the sonication time, ${ }^{35}$ reaching concentrations as high as $0.5 \mathrm{mg} / \mathrm{ml}$ after 300 hours. As with other solvents, the flakes were defect free. Using low boiling point solvents brings considerable advantages; for example, spray-casting of individual multilayer graphene flakes on surfaces was greatly simplified by using isopropanol.

We are currently working to scaleup solvent exfoliation of graphene. We now routinely produce up to a litre of graphene dispersion containing $\sim 1 \mathrm{~g}$ of graphene.

It is worth noting that a number of other groups have made considerable progress toward exfoliating graphene in solvents. ${ }^{36-47}$ This work has contributed greatly to our understanding of the dispersion process, our practical approach to dispersion and the number of solvents available to graphene researchers.

\section{Surfactant Stabilisation of graphene}


J.N. Coleman, Liquid exfoliation of defect free graphene, Accounts of Chemical Research

For many applications, exfoliation of graphene in solvents is undesirable due to a number of issues such as high boiling point, toxicity, incompatibility with other aspects of processing etc. To address this, we studied the possibility of exfoliating graphene in water in the presence of a surfactant stabiliser. We found that graphene can indeed be exfoliated by sonicating in aqueous solutions of surfactants such as sodium dodecylbenzenesulphonate and sodium cholate (figure 4A-C)). Although the fraction of monolayers is not as high as for solvent exfoliated graphene, the flake quality is good with little evidence of defects or oxides. ${ }^{48,49}$ As is the case for solvent exfoliation, the concentration can be increased dramatically by increasing the sonication time; approaching $0.3 \mathrm{mg} / \mathrm{ml}$ for 400 hours sonication. ${ }^{49}$ However, it is worth noting that the flake size does not decrease with sonication time as much as is observed for solvent exfoliation. This may be due to poor stress transfer during cavitation at the water/surfactant/graphene interface.

Like many colloidal systems, surfactant coated graphene is stabilised electrostatically against aggregation. Dissociation of the head group ions results in the formation of an electrical doublelayer. $^{50}$ The resultant Coulomb repulsion stabilises the sheets and is generally characterised by the zeta potential, the electrical potential at the edge of the layer of bound surfactant molecular ions. We studied a range of surfactants as stabilisers, finding zeta potentials in the range $\pm 25-65 \mathrm{mV} .{ }^{51}$ More interestingly, we found the dispersed concentration to scale with the square of the zeta potential. Because the electrostatic repulsive barrier stabilising the surfactant coated graphene also scales approximately with the square of the zeta potential, ${ }^{48}$ this suggests that the dispersed concentration is controlled by the size of this repulsive barrier (figure 4D).

It is worth noting that much progress has been made in surfactant exfoliation outside our group. ${ }^{52-56}$ This has led to a number of advances including using density gradient centrifugation to separate graphene flakes by thickness. ${ }^{52}$ In addition, it was recently shown that graphene can be exfoliated using adsorbed polymer as a stabiliser. ${ }^{57}$ Here the stabilisation mechanism is steric ${ }^{50}$ rather than electrostatic. We extended this method to show that graphene can be stabilised with polyurethane and polyvinylalcohol, a procedure that greatly facilitates composite formation. ${ }^{58,59}$

\section{Applications of liquid exfoliated graphene}

In our group, we have explored using solvent or surfactant exfoliated graphene in a number of applications. We have found that dispersions of graphene in solvents display a considerable broadband non-linear optical response when excited by nanosecond pulses. ${ }^{60}$ The optical transmission of $\sim 85 \%$ transparent dispersions falls to $\sim 48 \%$ as the pulse energy density is increased to $10 \mathrm{~J} / \mathrm{cm}^{2}$, suggesting the potential for optical limiting applications (figure $5 \mathrm{~A}$ ). 
J.N. Coleman, Liquid exfoliation of defect free graphene, Accounts of Chemical Research

We have also shown that pristine, solvent-exfoliated graphene shows potential as an electrode material in electrochemical sensors. ${ }^{42}$ We produced a novel ascorbic acid sensor based on pyrolysed photoresist films coated with graphene. This sensor was effective in the range 0.4 to $6.0 \mathrm{mM}$, with a $0.12 \mathrm{mM}$ detection limit (figure 5B). We extended this work to demonstrate sensing of $\beta$ nicotinamide adenine dinucleotide (NADH), a common enzyme. ${ }^{61}$ This works because the fouling which commonly hampers carbon-based NADH sensors does not occur at the pristine graphene surface.

We also attempted to prepare high performance transparent conductors from vacuum filtered films of surfactant-exfoliated graphene. However the results were disappointing; we achieved no better than transmittance of $76 \%$ coupled with a sheet resistance of $4 \mathrm{k} \Omega,{ }^{62}$ far below state-of-the-art materials (figure 5C). ${ }^{63}$ This prompted us to reanalyse a large number of published works, leading to the conclusion that the effect of inter-flake junction resistances means that films of liquid exfoliated graphene can never meet industry requirements for transparent conducting electrodes. ${ }^{64}$ They may however, be useful in applications where transparency is required but extremely low resistance is not necessary. However, we were able to show that graphene can be mixed with nanotubes to create hybrid films. ${ }^{65}$ These films have combinations of transmittance and sheet resistance significantly better than films of nanotubes or graphene alone. ${ }^{66}$

Due to its extremely high strength, ${ }^{10}$ one of the most promising applications of graphene is as a reinforcing material in polymer based composites. We found that as-prepared liquid exfoliated graphene can be used to effectively increase the low-strain-stress of elastomers ${ }^{58}$ but was less effective at reinforcing thermoplastics. We resolved this by using the size-selection technique described above to prepare dispersions of large aspect ratio flakes which could be mixed with polyvinylalcohol to give composites. We found very good reinforcement as characterised by a doubling in both modulus and strength on addition of $<0.5 \mathrm{wt} \%$ graphene (figure 5D). ${ }^{59}$ Detailed analysis showed these results to be exactly what would be expected from theory.

\section{Beyond Graphene}

A number of groups have shown that graphene can be chemically modified to produce compounds such as $\mathrm{CF}_{\mathrm{x}}$ and $\mathrm{CH}_{\mathrm{x}} \cdot{ }^{67-69}$ However, one can go beyond carbon altogether. Graphite is just one of a wide range of layered compounds. Exfoliation of such materials would give us a large number of new 2-dimensional materials with a wide range of properties. The first progress was reported recently with the solvent exfoliation of Boron Nitride. ${ }^{70}$ Perhaps more interesting is the family of transition metal dichalcogenides, which contains over 40 members with compositions such as $\mathrm{MoS}_{2}, \mathrm{WSe}_{2}, \mathrm{NiTe}_{2}$ etc. These materials can be metallic or semiconducting with a range of bandgaps depending on composition. Recently, we showed that a range of these materials can be 
J.N. Coleman, Liquid exfoliation of defect free graphene, Accounts of Chemical Research

exfoliated in large quantities either in solvents or using surfactants. ${ }^{60,71}$ Importantly, dispersions of these materials can be mixed with dispersions of nanotubes or graphene to produce highly conductive hybrids. We anticipate that these hybrids will be useful in a range of areas from Li ion batteries to thermoelectrics.

Concluding remarks

As the potential applications of graphene multiply, it will become more and more important to be able to produce defect-free graphene in large quantities. In this report, we have shown that this can be achieved by sonication of graphite in certain liquids. The ultrasound results in the exfoliation and cutting of the graphite crystallites to give graphene nanosheets. These sheets are then stabilised, either by interaction with the solvent or by the presence of adsorbed surfactant or polymer. Raman and XPS spectroscopy show the flakes to be defect and oxide free. While we have shown these flakes to be useful in a number of applications, we believe the next decade will see an explosion in the usage of these materials in a wide range of applications.

\section{Biography}

Jonathan Coleman was born in Dublin, Ireland in 1973. He received both his B.A (1995) and Ph.D. (2000) in Physics from Trinity College Dublin. He remained at Trinity as a postdoc, lecturer and associate professor. He is now Professor of Chemical Physics and a Principal Investigator in the Centre for Research on Adaptive nanostructures and Nanodevices (CRANN). His research interests focus on the processing of nanomaterials in liquids for a range of applications.

\section{Acknowledgments}

I would like to acknowledgment SFI funding under the PI award scheme, contract number 07/IN.1/I1772. In addition, I must thank all those within my group who contributed to this work. It is impossible to mention you all but special thanks must go to Dr Yenny Hernandez and Dr Valeria Nicolosi.

Supporting Information Available: List of solvents and surfactants. This information is available free of charge via the Internet at http://pubs.acs.org/. 
J.N. Coleman, Liquid exfoliation of defect free graphene, Accounts of Chemical Research
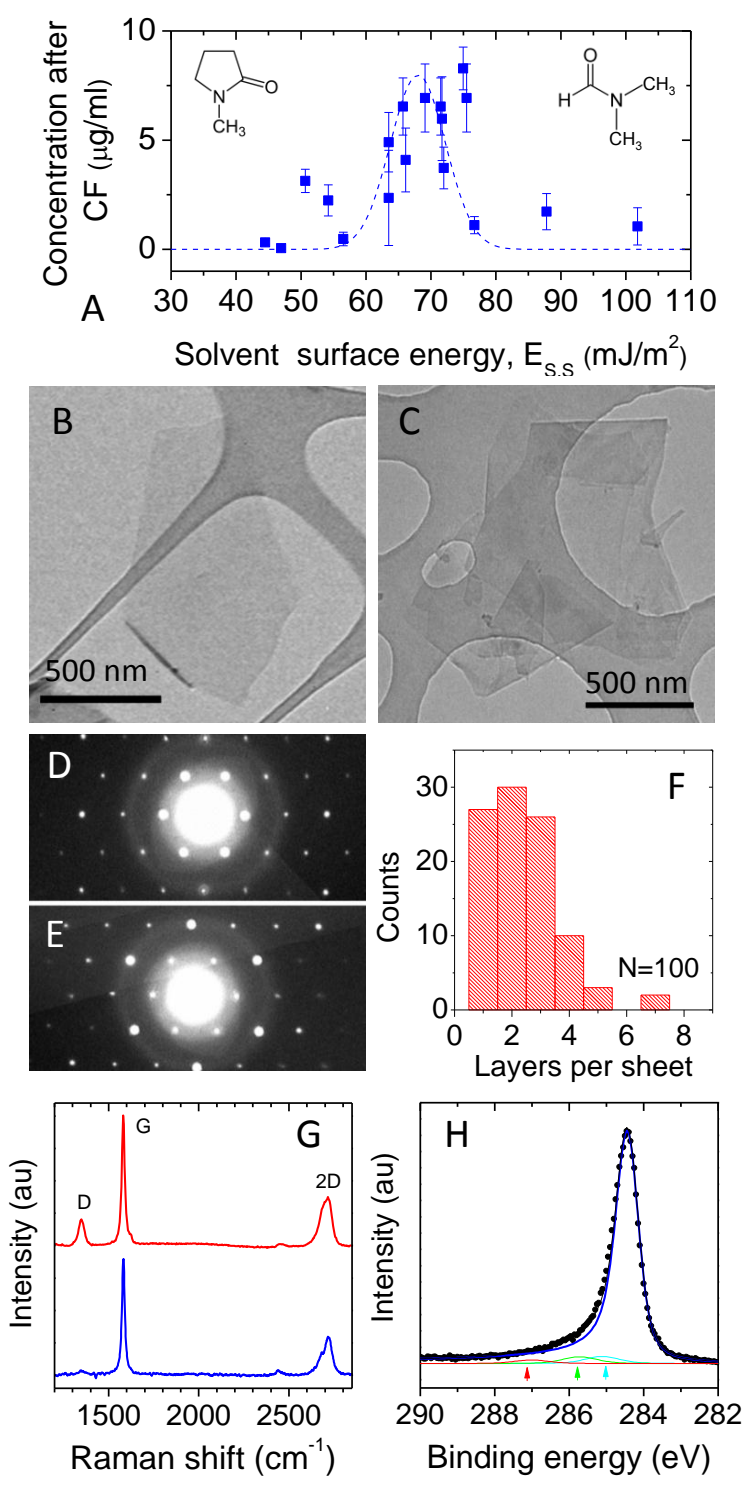

Figure 1: A) Dispersed graphene concentration as a function of solvent surface energy. Insets: Structures of NMP (left) and DMF (right). B) and C) TEM images of graphene monolayer and multilayer respectively. D) and E) Electron diffraction patterns of graphene monolayer and multilayer respectively. F) Histogram showing the distribution of graphene layer thicknesses. G) Raman spectra of (top) a small flake and (bottom) a larger flake. H) C1s XPS spectrum of a thin film of graphene flakes. The contributions marked by arrows are due to the solvent. 
J.N. Coleman, Liquid exfoliation of defect free graphene, Accounts of Chemical Research
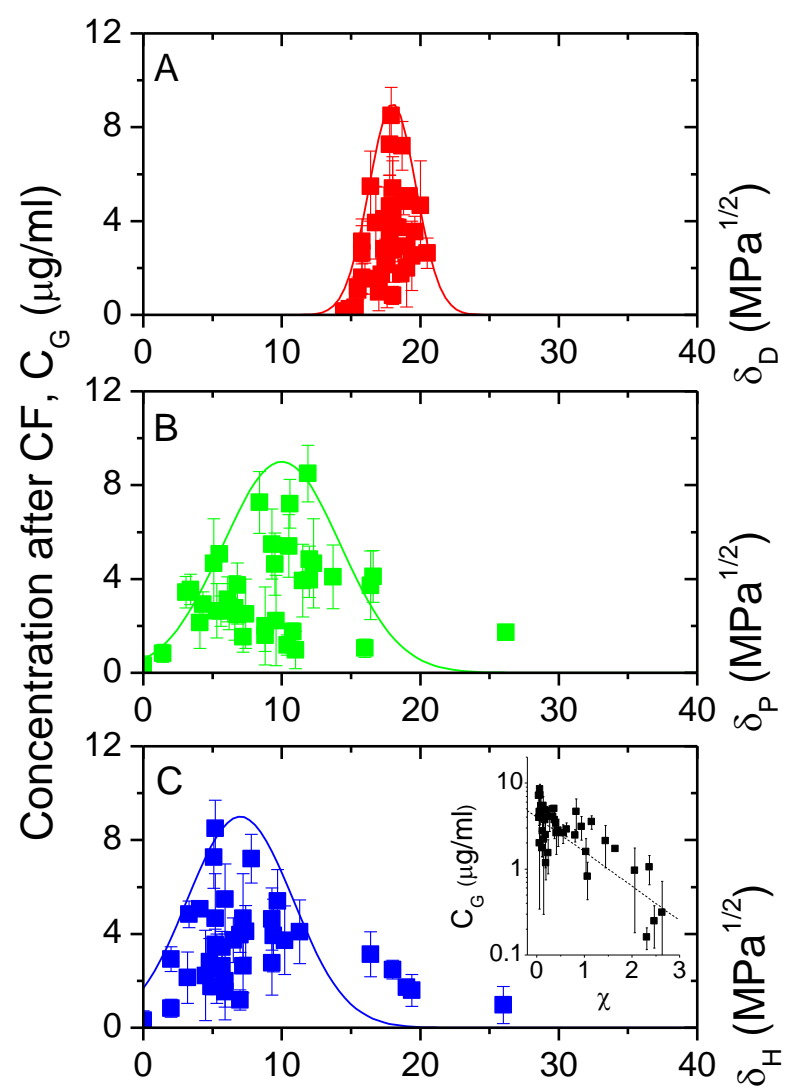

Figure 2: Dispersed graphene concentration as a function of A) dispersive, B) polar and C) Hydrogen bonding Hansen solubility parameters. Inset: Log of dispersed concentration as a function of FloryHuggins parameter. Linearity (dashed line) is expected from solubility theory. Note all solvents included in this data set are listed in the supporting information. 
J.N. Coleman, Liquid exfoliation of defect free graphene, Accounts of Chemical Research
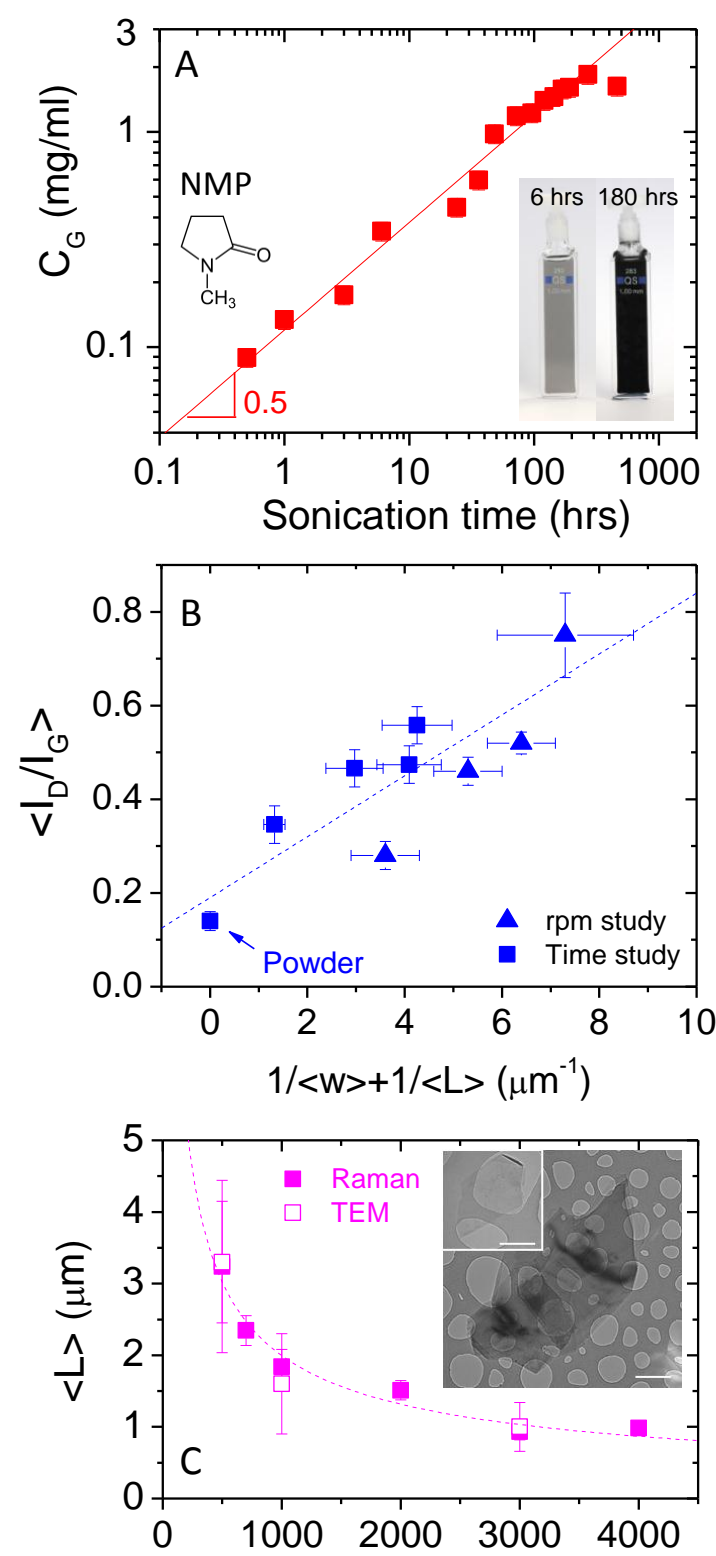

Final centrifugation rate, $\omega(\mathrm{rpm})$

Figure 3: A) Concentration of dispersed graphene in $\mathrm{N}$-methyl pyrrolidone as a function of sonication time. The line illustrates square root behaviour. Inset: Photos of dispersions after two different sonication times and the structure of the solvent. B) Defect content as represented by the Raman D:G ratio plotted versus flake length to area ratio. C) Length of size selected flakes plotted as a function of final centrifugation rate. Inset: Flakes found after final centrifugation at $3000 \mathrm{rpm}$ (small flake) and $5000 \mathrm{rpm}$ (large flakes). In each case, the scales are identical, scale bar: $500 \mathrm{~nm}$. 
J.N. Coleman, Liquid exfoliation of defect free graphene, Accounts of Chemical Research

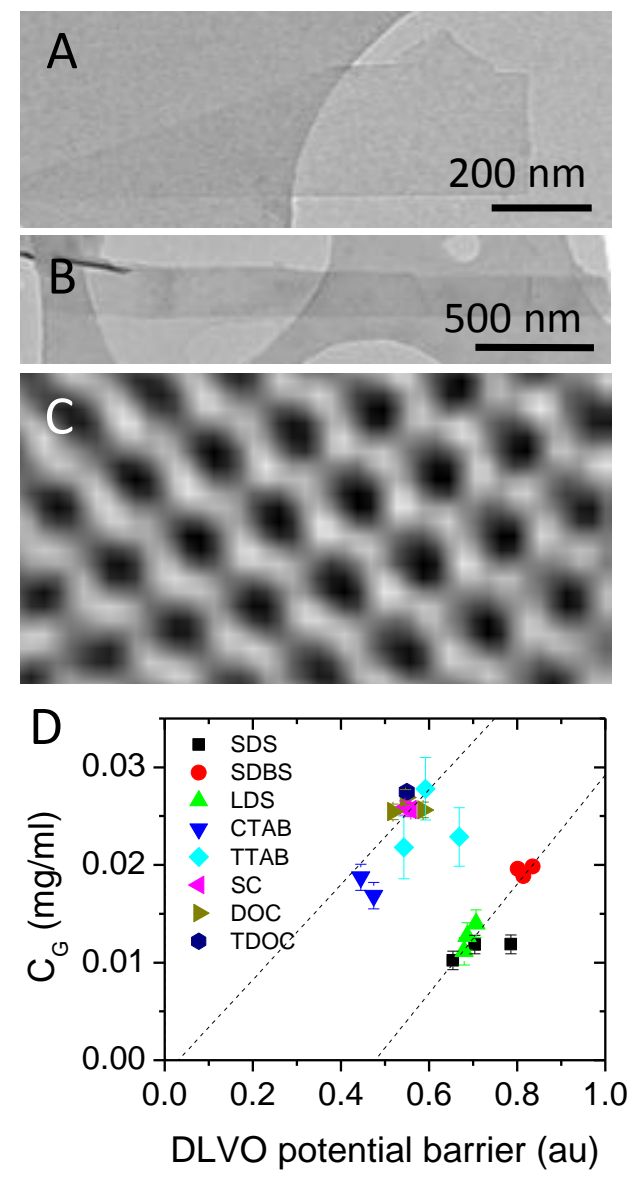

Figure 4: A) and B) TEM images of surfactant exfoliated flakes. C) Digitally filtered high resolution image of a surfactant exfoliated flake. D) Dispersed concentration as a function of electrostatic potential barrier. The full names and structures of the surfactants are given in the supporting information. 
J.N. Coleman, Liquid exfoliation of defect free graphene, Accounts of Chemical Research

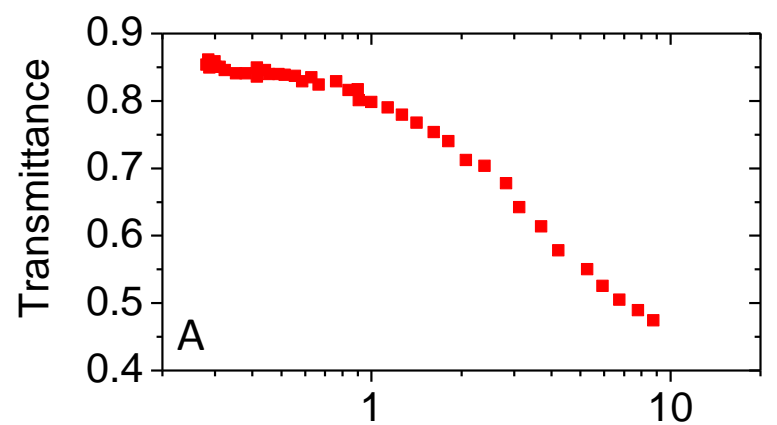

Pulse energy density $\left(\mathrm{J} / \mathrm{cm}^{2}\right)$
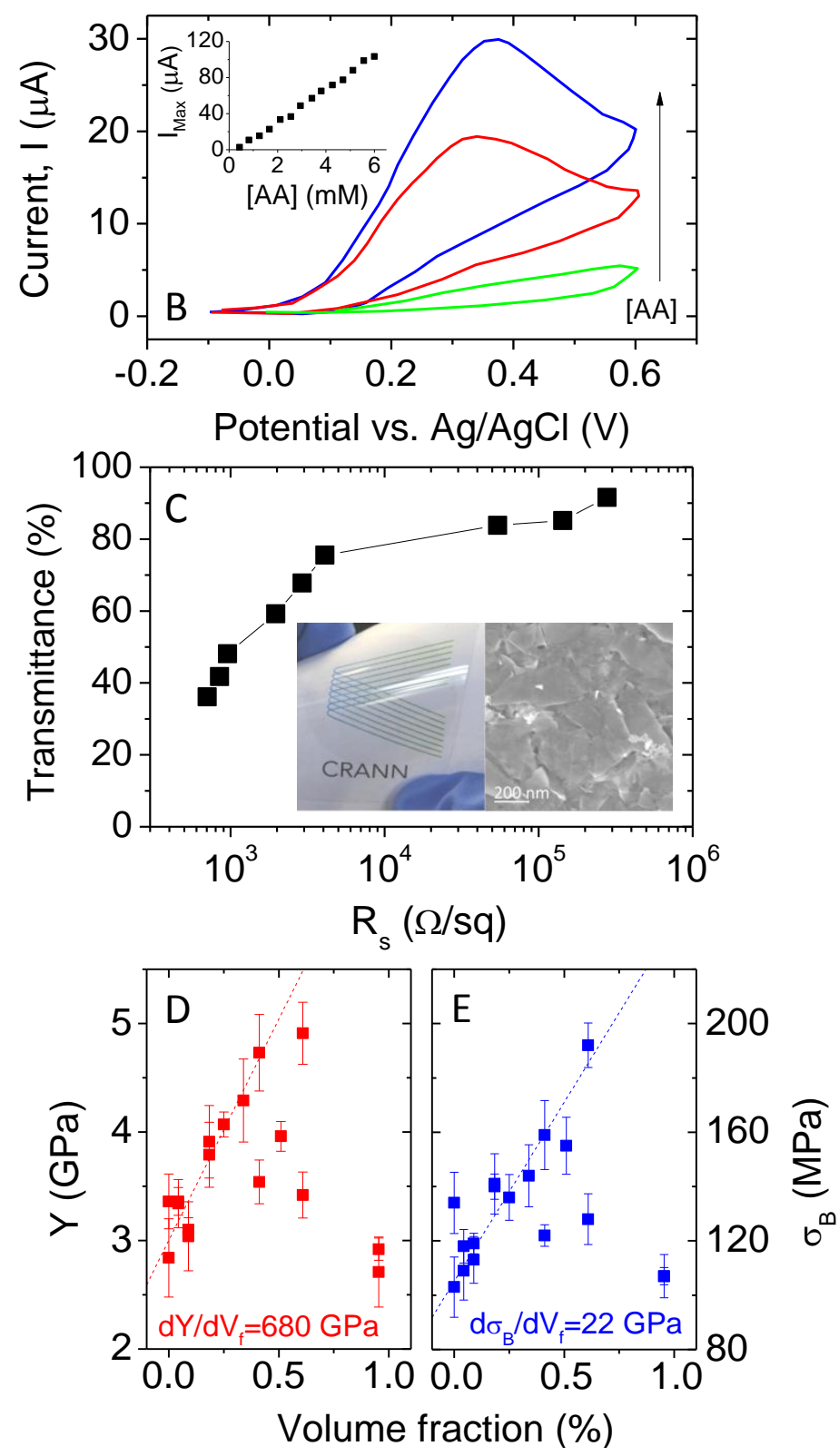

Figure 5: A) measured transmittance as a function of pulse energy density for a dispersion of graphene in dimethylacetamide. B) Cyclic voltammograms of graphene electrodes in ascorbic acid (AA) solutions at a range of concentrations. Inset: Electro-catalytic peak current against ascorbic acid concentration. C) Transmittance versus sheet resistance for graphene thin films. Inset: Photo of a transparent conducting film (left) and SEM image of the film surface (right). D) Young's modulus 
and E) strength of graphene-polyvinylalcohol composites as a function of graphene volume fraction. The slopes of the linear regions are given.

(1) The Cambridge Ancient History, Volume 3, Part 1; Boardman, J.; Edwards, I. E. S.; Hammond, N. G. L.; Sollberger, E., Eds.; Cambridge University Press: Cambridge, 2008.

(2) Bernal, J. D.: The Structure of Graphite. Proceedings of the Royal Society of London, Series A 1924, 106, 749 .

(3) Geim, A. K.: Graphene: Status and Prospects. Science 2009, 324, 1530-1534.

(4) Geim, A. K.; Novoselov, K. S.: The rise of graphene. Nat. Mater. 2007, 6, 183-191.

(5) Wallace, P. R.: The Band Theory of Graphite. Physical Review 1947, 71, 622-634.

(6) Mermin, N. D.: Crystalline Order in Two Dimensions. Physical Review 1968, 176, 250-254.

(7) Boehm, H. P.; Clauss, A.; Fischer, G.; Hofmann, U.: Surface properties of extremely thin graphite lamellae. Proceedings of the Fifth Conference on Carbon 1962, Pergamon Press, 73-80.

(8) Lu, X. K.; Yu, M. F.; Huang, H.; Ruoff, R. S.: Tailoring graphite with the goal of achieving single sheets. Nanotechnology 1999, 10, 269-272.

(9) Novoselov, K. S.; Geim, A. K.; Morozov, S. V.; Jiang, D.; Zhang, Y.; Dubonos, S. V.; Grigorieva, I. V.; Firsov, A. A.: Electric field effect in atomically thin carbon films. Science 2004, 306, 666669.

(10) Lee, C.; Wei, X. D.; Kysar, J. W.; Hone, J.: Measurement of the elastic properties and intrinsic strength of monolayer graphene. Science 2008, 321, 385-388.

(11) Bae, S.; Kim, H.; Lee, Y.; Xu, X. F.; Park, J. S.; Zheng, Y.; Balakrishnan, J.; Lei, T.; Kim, H. R.; Song, Y. I.; Kim, Y. J.; Kim, K. S.; Ozyilmaz, B.; Ahn, J. H.; Hong, B. H.; Iijima, S.: Roll-to-roll production of 30-inch graphene films for transparent electrodes. Nature Nanotechnology 2010, 5, 574-578.

(12) Berger, C.; Song, Z. M.; Li, X. B.; Wu, X. S.; Brown, N.; Naud, C.; Mayo, D.; Li, T. B.; Hass, J.; Marchenkov, A. N.; Conrad, E. H.; First, P. N.; de Heer, W. A.: Electronic confinement and coherence in patterned epitaxial graphene. Science 2006, 312, 1191-1196.

(13) Kim, K. S.; Zhao, Y.; Jang, H.; Lee, S. Y.; Kim, J. M.; Kim, K. S.; Ahn, J.-H.; Kim, P.; Choi, J.-Y.; Hong, B. H.: Large-scale pattern growth of graphene films for stretchable transparent electrodes. Nature 2009.

(14) Li, X. S.; Cai, W. W.; An, J. H.; Kim, S.; Nah, J.; Yang, D. X.; Piner, R.; Velamakanni, A.; Jung, I.; Tutuc, E.; Banerjee, S. K.; Colombo, L.; Ruoff, R. S.: Large-Area Synthesis of High-Quality and Uniform Graphene Films on Copper Foils. Science 2009, 324, 1312-1314.

(15) Kuilla, T.; Bhadra, S.; Yao, D. H.; Kim, N. H.; Bose, S.; Lee, J. H.: Recent advances in graphene based polymer composites. Progress in Polymer Science 2010, 35, 1350-1375.

(16) Ruoff, R.: Calling all chemists. Nature Nanotechnology 2008, 3, 10-11.

(17) Stankovich, S.; Dikin, D. A.; Piner, R. D.; Kohlhaas, K. A.; Kleinhammes, A.; Jia, Y.; Wu, Y.; Nguyen, S. T.; Ruoff, R. S.: Synthesis of graphene-based nanosheets via chemical reduction of exfoliated graphite oxide. Carbon 2007, 45, 1558-1565.

(18) Zhu, Y. W.; Murali, S.; Cai, W. W.; Li, X. S.; Suk, J. W.; Potts, J. R.; Ruoff, R. S.: Graphene and Graphene Oxide: Synthesis, Properties, and Applications. Advanced Materials 2010, 22, 3906-3924.

(19) Coleman, J. N.: Liquid-Phase Exfoliation of Nanotubes and Graphene. Advanced Functional Materials 2009, 19, 3680-3695.

(20) Bergin, S. D.; Nicolosi, V.; Streich, P. V.; Giordani, S.; Sun, Z. Y.; Windle, A. H.; Ryan, P.; Niraj, N. P. P.; Wang, Z. T. T.; Carpenter, L.; Blau, W. J.; Boland, J. J.; Hamilton, J. P.; Coleman, J. N.: Towards solutions of single-walled carbon nanotubes in common solvents. Advanced Materials 2008, 20, 1876-+.

(21) Bergin, S. D.; Sun, Z. Y.; Rickard, D.; Streich, P. V.; Hamilton, J. P.; Coleman, J. N.: Multicompenent solubility parameters for single walled carbon nanotube-solvent mixtures. ACS Nano 2009, 3 , $2340-2350$.

(22) Hughes, J. M.; Aherne, D.; Bergin, S. D.; Streich, P. V.; Hamilton, J. P.; Coleman, J. N.: Using solution thermodynamics to describe the dispersion of carbon nanotubes in organic solvents. Langmuir 2011, accepted.

(23) Hernandez, Y.; Nicolosi, V.; Lotya, M.; Blighe, F. M.; Sun, Z. Y.; De, S.; McGovern, I. T.; Holland, B.; Byrne, M.; Gun'ko, Y. K.; Boland, J. J.; Niraj, P.; Duesberg, G.; Krishnamurthy, S.; Goodhue, R.; 
Hutchison, J.; Scardaci, V.; Ferrari, A. C.; Coleman, J. N.: High-yield production of graphene by liquid-phase exfoliation of graphite. Nature Nanotechnology 2008, 3, 563-568.

(24) Lyklema, J.: The surface tension of pure liquids - Thermodynamic components and corresponding states. Colloids and Surfaces a-Physicochemical and Engineering Aspects 1999, 156, 413-421.

(25) Wang, S. R.; Zhang, Y.; Abidi, N.; Cabrales, L.: Wettability and Surface Free Energy of Graphene Films. Langmuir 2009, 25, 11078-11081.

(26) Khan, U.; O'Neill, A.; Lotya, M.; De, S.; Coleman, J. N.: High-Concentration Solvent Exfoliation of Graphene. Small 2010, 6, 864-871.

(27) Meyer, J. C.; Geim, A. K.; Katsnelson, M. I.; Novoselov, K. S.; Obergfell, D.; Roth, S.; Girit, C.; Zettl, A.: On the roughness of single- and bi-layer graphene membranes. Solid State Commun. 2007, 143, 101-109.

(28) Horiuchi, S.; Gotou, T.; Fuijwara, M.; Sotoaka, R.; Hirata, M.; Kimoto, K.; Asaka, T.; Yokosawa, T.; Matsui, Y.; Watanabe, K.; Sekita, M.: Carbon nanofilm with a new structure and property. Japanese Journal of Applied Physics Part 2-Letters 2003, 42, L1073-L1076.

(29) Ferrari, A. C.; Meyer, J. C.; Scardaci, V.; Casiraghi, C.; Lazzeri, M.; Mauri, F.; Piscanec, S.; Jiang, D.; Novoselov, K. S.; Roth, S.; Geim, A. K.: Raman spectrum of graphene and graphene layers. Phys. Rev. Lett. 2006, 97.

(30) Hansen, C. M.: Hansen Solubility Parameters - A User's Handbook; CRC Press, 2007.

(31) Hernandez, Y.; Lotya, M.; Rickard, D.; Bergin, S. D.; Coleman, J. N.: Measurement of Multicomponent Solubility Parameters for Graphene Facilitates Solvent Discovery. Langmuir 2010, 26, 32083213.

(32) Hennrich, F.; Krupke, R.; Arnold, K.; Rojas Stutz, J. A.; Lebedkin, S.; Koch, T.; Schimmel, T.; Kappes, M. M.: The Mechanism of Cavitation-Induced Scission of Single-Walled Carbon Nanotubes. The Journal of Physical Chemistry B 2007, 111, 1932-1937.

(33) Khan, U.; Porwal, H.; O’Neill, A.; Nawaz, K.; May, P.; Coleman, J. N.: Solvent-Exfoliated Graphene at Extremely High Concentration. Langmuir 2011, 27, 9077-9082.

(34) Gong, L.; Kinloch, I. A.; Young, R. J.; Riaz, I.; Jalil, R.; Novoselov, K. S.: Interfacial Stress Transfer in a Graphene Monolayer Nanocomposite. Advanced Materials 2010, in press.

(35) O'Neill, A.; Khan, U.; Nirmalraj, P. N.; Boland, J. J.; Coleman, J. N.: Graphene Dispersion and Exfoliation in Low Boiling Point Solvents. The Journal of Physical Chemistry C 2011, 115, 5422-5428.

(36) Blake, P.; Brimicombe, P. D.; Nair, R. R.; Booth, T. J.; Jiang, D.; Schedin, F.; Ponomarenko, L. A.; Morozov, S. V.; Gleeson, H. n. F.; Hill, E. W.; Geim, A. K.; Novoselov, K. S.: Graphene-Based Liquid Crystal Device. Nano Letters 2008, 8, 1704-1708.

(37) Bourlinos, A. B.; Georgakilas, V.; Zboril, R.; Steriotis, T. A.; Stubos, A. K.: Liquid-Phase Exfoliation of Graphite Towards Solubilized Graphenes. Small 2009, 5, 1841-5.

(38) Hamilton, C. E.; Lomeda, J. R.; Sun, Z. Z.; Tour, J. M.; Barron, A. R.: High-Yield Organic Dispersions of Unfunctionalized Graphene. Nano Letters 2009, 9, 3460-3462.

(39) Hasan, T.; Torrisi, F.; Sun, Z.; Popa, D.; Nicolosi, V.; Privitera, G.; Bonaccorso, F.; Ferrari, A. C.: Solution-phase exfoliation of graphite for ultrafast photonics. Physica Status Solidi B-Basic Solid State Physics 2010, 247, 2953-2957.

(40) Choi, E. Y.; Choi, W. S.; Lee, Y. B.; Noh, Y. Y.: Production of graphene by exfoliation of graphite in a volatile organic solvent. Nanotechnology 2011, 22.

(41) Khan, U.; O’Neill, A.; Porwal, H.; May, P.; Nawaz, K.; Coleman, J. N.: Size selection of dispersed, exfoliated graphene flakes by controlled centrifugation. Carbon 2012, 50, 470-475.

(42) Keeley, G. P.; O'Neill, A.; McEvoy, N.; Peltekis, N.; Coleman, J. N.; Duesberg, G. S.: Electrochemical ascorbic acid sensor based on DMF-exfoliated graphene. J. Mater. Chem. 2010, 20, 78647869.

(43) Liang, Y. T.; Hersam, M. C.: Highly Concentrated Graphene Solutions via Polymer Enhanced Solvent Exfoliation and Iterative Solvent Exchange. Journal of the American Chemical Society 2010, 132, 17661-17663.

(44) Nuvoli, D.; Valentini, L.; Alzari, V.; Scognamillo, S.; Bon, S. B.; Piccinini, M.; Illescas, J.; Mariani, A.: High concentration few-layer graphene sheets obtained by liquid phase exfoliation of graphite in ionic liquid. J. Mater. Chem. 2011, 21, 3428-3431.

(45) Zhang, X. Y.; Coleman, A. C.; Katsonis, N.; Browne, W. R.; van Wees, B. J.; Feringa, B. L.: Dispersion of graphene in ethanol using a simple solvent exchange method. Chemical Communications 2010, 46, 7539-7541. 
(46) Alzari, V.; Nuvoli, D.; Scognamillo, S.; Piccinini, M.; Gioffredi, E.; Malucelli, G.; Marceddu, S.; Sechi, M.; Sanna, V.; Mariani, A.: Graphene-containing thermoresponsive nanocomposite hydrogels of poly(N-isopropylacrylamide) prepared by frontal polymerization. J. Mater. Chem. 2011, 21, 8727-8733.

(47) Shih, C. J.; Lin, S. C.; Strano, M. S.; Blankschtein, D.: Understanding the Stabilization of Liquid-Phase-Exfoliated Graphene in Polar Solvents: Molecular Dynamics Simulations and Kinetic Theory of Colloid Aggregation. Journal of the American Chemical Society 2010, 132, 14638-14648.

(48) Lotya, M.; Hernandez, Y.; King, P. J.; Smith, R. J.; Nicolosi, V.; Karlsson, L. S.; Blighe, F. M.; De, S.; Wang, Z.; McGovern, I. T.; Duesberg, G. S.; Coleman, J. N.: Liquid Phase Production of Graphene by Exfoliation of Graphite in Surfactant/Water Solutions. Journal of the American Chemical Society 2009, 131, 3611-3620.

(49) Lotya, M.; King, P. J.; Khan, U.; De, S.; Coleman, J. N.: High-Concentration, SurfactantStabilized Graphene Dispersions. ACS Nano 2010, 4, 3155-3162.

(50) Israelachvili, J.: Intermolecular and Surface Forces; Second Edition ed.; Academic press,

1991.

(51) Smith, R. J.; Lotya, M.; Coleman, J. N.: The importance of repulsive potential barriers for the dispersion of graphene using surfactants. New Journal of Physics 2010, 12, 125008.

(52) Green, A. A.; Hersam, M. C.: Solution Phase Production of Graphene with Controlled Thickness via Density Differentiation. Nano Letters 2009, 9, 12.

(53) Hao, R.; Qian, W.; Zhang, L. H.; Hou, Y. L.: Aqueous dispersions of TCNQ-anion-stabilized graphene sheets. Chemical Communications 2008, 6576-6578.

(54) Vadukumpully, S.; Paul, J.; Valiyaveettil, S.: Cationic surfactant mediated exfoliation of graphite into graphene flakes. Carbon 2009, 47, 3288-3294.

(55) Lin, S. C.; Shih, C. J.; Strano, M. S.; Blankschtein, D.: Molecular Insights into the Surface Morphology, Layering Structure, and Aggregation Kinetics of Surfactant-Stabilized Graphene Dispersions. Journal of the American Chemical Society 2011, 133, 12810-12823.

(56) Wei, W.; Lu, W.; Yang, Q. H.: High-concentration graphene aqueous suspension and a membrane self-assembled at the liquid-air interface. New Carbon Materials 2011, 26, 36-40.

(57) Bourlinos, A. B.; Georgakilas, V.; Zboril, R.; Steriotis, T. A.; Stubos, A. K.; Trapalis, C.: Aqueous-phase exfoliation of graphite in the presence of polyvinylpyrrolidone for the production of watersoluble graphenes. Solid State Commun. 2009, 149, 2172-2176.

(58) Khan, U.; May, P.; O'Neill, A.; Coleman, J. N.: Development of stiff, strong, yet tough composites by the addition of solvent exfoliated graphene to polyurethane. Carbon 2010, 48, 4035-4041.

(59) May, P.; Khan, U.; O'Neill, A.; Coleman, J. N.: Approaching the theoretical limit for reinforcing polymers with graphene. J. Mater. Chem. 2012, web publication.

(60) Smith, R. J.; King, P. J.; Lotya, M.; Wirtz, C.; Khan, U.; De, S.; O'Neill, A.; Duesberg, G. S.; Grunlan, J. C.; Moriarty, G.; Chen, J.; Wang, J. Z.; Minett, A. I.; Nicolosi, V.; Coleman, J. N.: Large-Scale Exfoliation of Inorganic Layered Compounds in Aqueous Surfactant Solutions. Advanced Materials 2011, 23, 3944-+.

(61) Keeley, G. P.; O'Neill, A.; Holzinger, M.; Cosnier, S.; Coleman, J. N.; Duesberg, G. S.: DMFexfoliated graphene for electrochemical NADH detection. Physical Chemistry Chemical Physics 2011, 13, 7747-7750.

(62) De, S.; King, P. J.; Lotya, M.; O'Neill, A.; Doherty, E. M.; Hernandez, Y.; Duesberg, G. S.; Coleman, J. N.: Flexible, Transparent, Conducting Films of Randomly Stacked Graphene from SurfactantStabilized, Oxide-Free Graphene Dispersions. Small 2010, 6, 458-464.

(63) Scardaci, V.; Coull, R.; Lyons, P. E.; Rickard, D.; Coleman, J. N.: Spray Deposition of Highly Transparent, Low-Resistance Networks of Silver Nanowires over Large Areas. Small 2011, 7, 2621-2628.

(64) De, S.; Coleman, J. N.: Are There Fundamental Limitations on the Sheet Resistance and Transmittance of Thin Graphene Films? ACS Nano 2010, 4, 2713-2720.

(65) Khan, U.; O'Connor, I.; Gun'ko, Y. K.; Coleman, J. N.: The preparation of hybrid films of carbon nanotubes and nano-graphite/graphene with excellent mechanical and electrical properties. Carbon 2010, 48, 2825-2830.

(66) King, P. J.; Khan, U.; Lotya, M.; De, S.; Coleman, J. N.: Improvement of Transparent Conducting Nanotube Films by Addition of Small Quantities of Graphene. ACS Nano 2010, 4, 4238-4246.

(67) Nair, R. R.; Ren, W. C.; Jalil, R.; Riaz, I.; Kravets, V. G.; Britnell, L.; Blake, P.; Schedin, F.; Mayorov, A. S.; Yuan, S. J.; Katsnelson, M. I.; Cheng, H. M.; Strupinski, W.; Bulusheva, L. G.; Okotrub, A. 
V.; Grigorieva, I. V.; Grigorenko, A. N.; Novoselov, K. S.; Geim, A. K.: Fluorographene: A Two-Dimensional Counterpart of Teflon. Small 2010, 6, 2877-2884.

(68) Zboril, R.; Karlicky, F.; Bourlinos, A. B.; Steriotis, T. A.; Stubos, A. K.; Georgakilas, V.;

Safarova, K.; Jancik, D.; Trapalis, C.; Otyepka, M.: Graphene Fluoride: A Stable Stoichiometric Graphene Derivative and its Chemical Conversion to Graphene. Small 2010, 6, 2885-2891.

(69) Elias, D. C.; Nair, R. R.; Mohiuddin, T. M. G.; Morozov, S. V.; Blake, P.; Halsall, M. P.;

Ferrari, A. C.; Boukhvalov, D. W.; Katsnelson, M. I.; Geim, A. K.; Novoselov, K. S.: Control of Graphene's Properties by Reversible Hydrogenation: Evidence for Graphane. Science 2009, 323, 610-613.

(70) Zhi, C. Y.; Bando, Y.; Tang, C. C.; Kuwahara, H.; Golberg, D.: Large-Scale Fabrication of Boron Nitride Nanosheets and Their Utilization in Polymeric Composites with Improved Thermal and Mechanical Properties. Advanced Materials 2009, 21, 2889-+.

(71) Coleman, J. N.; Lotya, M.; O'Neill, A.; Bergin, S. D.; King, P. J.; Khan, U.; Young, K.; Gaucher, A.; De, S.; Smith, R. J.; Shvets, I. V.; Arora, S. K.; Stanton, G.; Kim, H. Y.; Lee, K.; Kim, G. T.; Duesberg, G. S.; Hallam, T.; Boland, J. J.; Wang, J. J.; Donegan, J. F.; Grunlan, J. C.; Moriarty, G.; Shmeliov, A.; Nicholls, R. J.; Perkins, J. M.; Grieveson, E. M.; Theuwissen, K.; McComb, D. W.; Nellist, P. D.; Nicolosi, V.: Two-Dimensional Nanosheets Produced by Liquid Exfoliation of Layered Materials. Science 2011, 331, 568-571. 